\title{
Interferon is associated with improved survival for node-positive cutaneous melanoma: a single-institution experience
}

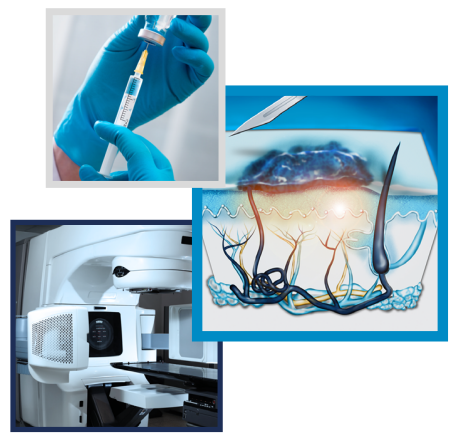

\author{
Daniel E Oliver*, ${ }^{*}, 1$, Vernon K Sondak ${ }^{\dagger, 2,3}$, Tobin Strom ${ }^{1,4}$, Jonathan S Zager ${ }^{2,3}$, Arash O \\ Naghavi1,2, Amod Sarnaik 2,3, Jane L Messina 2,3,5,6, Jimmy J Caudell1,2, Andy M Trotti ${ }^{1,2}$, \\ Javier F Torres-Roca ${ }^{1,2}$, Nikhil I Khushalani ${ }^{\ddagger 2,3}$ \& Louis B Harrison ${ }^{\ddagger 1,2}$ \\ ${ }^{1}$ Department of Radiation Oncology, Moffitt Cancer Center, Tampa, FL 33612, USA \\ ${ }^{2}$ Department of Oncologic Sciences, University of South Florida Morsani College of Medicine, Tampa, FL 33612, USA \\ ${ }^{3}$ Department of Cutaneous Oncology, Moffitt Cancer Center, Tampa, FL 33612, USA \\ ${ }^{4}$ Department of Radiation Oncology, University of Texas Southwestern, Dallas, TX 75390, USA \\ ${ }^{5}$ Department of Anatomic Pathology, Moffitt Cancer Center, Tampa, FL 33612, USA \\ ${ }^{6}$ Departments of Pathology \& Cell Biology \& Dermatology, University of South Florida Morsani College of Medicine, Tampa, FL \\ 33612 , USA \\ *Author for correspondence: Tel.: +01 813745 5134; daniel.oliver@moffit.org \\ †These authors contributed equally \\ ¥These authors contributed equally
}

\section{Summary points}

\section{Primary end points}

- Among node-positive patients, adjuvant interferon was associated with $14 \%$ increase in relapse-free survival, $12 \%$ increase in distant metastasis-free survival and $16 \%$ increase in overall survival at 5 years.

Predictors of worse outcome

- Factors predicting for worse outcomes on multivariable analysis included male sex, T3/4 tumor status, $\mathrm{N} 1 \mathrm{~b} / \mathrm{N} 2 \mathrm{~b} / \mathrm{N} 3$ nodal stage, extranodal extension, nodal metastasis size $>2 \mathrm{~cm}$ and delivery of regional radiotherapy.

\section{Age-matched analysis}

- Improvement in all primary end points remained apparent on an exploratory age-matched comparison of those patients treated with (median age: 61 years) versus without (median age: 63 years) adjuvant interferon.

Conclusion

- Most randomized trials addressing the role of adjuvant interferon were performed before sentinel lymph node biopsy, and had suboptimal regional control.

- The majority of patients in the current study had nodal disease detected by sentinel lymph node biopsy compared with the predominant bulky nodal disease seen in these early trials.

- In this setting, our findings show a substantial and clinically relevant improvement in both distant metastasis-free survival and overall survival.

- Significant advances in the adjuvant therapy landscape have been made with CTLA-4 blockade and anti-PD1 therapy; however, reports of severe toxicity warrant careful patient selection.

- Although adjuvant anti-PD1 therapy has emerged as the current standard in the USA, no prospective data are yet available showing a survival benefit compared with interferon or ipilimumab. The current findings provide context for comparison in areas where newer systemic therapies have not yet been widely adopted.

Aim: We assessed the role of adjuvant interferon on relapse-free survival (RFS), distant metastasis-free survival (DMFS) and overall survival (OS) in node-positive melanoma patients. Methods: We retrospectively reviewed 385 node-positive patients without distant metastatic disease treated from 1998 to 2015 . The surgery was therapeutic lymph node dissection (LND, $n=86$ ) or sentinel lymph node biopsy \pm completion LND $(n=270) .128$ patients $(33.2 \%)$ received adjuvant interferon. Results: After a median follow-up of 70 months, interferon was associated with improved RFS (hazard ratio [HR]: 0.55; $p<0.001$ ), DMFS (HR: 0.59; $p<0.001$ ) and OS (HR: 0.61; $p=0.003)$, controlling for tumor and nodal stage, node size, sex, primary site, adjuvant therapy and extracapsular extension. In an exploratory age-matched comparison of patients treated with $(n=67)$ and without $(n=233)$ adjuvant immunotherapy, interferon still showed improved RFS, 
DMFS and OS. Conclusion: Adjuvant interferon appears to improve OS among node-positive melanoma patients in a modern experience, providing context for comparison in the adjuvant therapy landscape.

First draft submitted: 6 October 2017; Accepted for publication: 22 February 2017; Published online: 09 April 2018

Keywords: cutaneous • immunotherapy • interferon • melanoma • survival

Cutaneous melanoma rates are increasing worldwide and have doubled in the USA over the past 30 years [1]. Stage III (node-positive) disease exhibits diverse outcomes, with sentinel node detected disease having a much better prognosis than that which is clinically palpable $[2,3]$. For example, 5-year survival rates for stage III melanoma range from 40 to $78 \%$, depending on the extent of nodal involvement [2]. Since sentinel lymph node biopsy (SLNB) has become standard practice, there has been a corresponding shift in the stage III patient population [4]. Micrometastatic disease is being detected more frequently, and thus, more stage III patients are presenting with disease in a sentinel lymph node (LN) than with bulky lymphadenopathy.

The search for an optimal adjuvant therapy to prevent the development of systemic disease has been an area of intense investigation. Multiple clinical trials show that adjuvant interferon improves relapse-free survival (RFS) [5-8], and other studies suggest a benefit for overall survival (OS) as well [9,10]. Most studies, however, were done before SLNB and often had suboptimal regional control $[6,7,11]$. This may have impacted the ability to detect a sustained OS benefit. The toxicity profile of interferon, in combination with its small OS benefit, has led to increasing hesitancy among oncologists in recommending interferon as standard adjuvant treatment for stage III melanoma. This is reflected in the current NCCN guidelines [12]. Recent landmark trials have shown promise in the adjuvant use of CTLA4-blockade [13,14] and anti-PD therapy [15]; however, reports of severe toxicity warrant careful patient selection and no prospective trial has yet shown a survival benefit compared with interferon. Some contemporary clinical trials continue to use adjuvant interferon as the comparator arm, while others do not.

In an institutional analysis of our node-positive melanoma patients, we previously reviewed the association of postoperative therapy with regional control [16]. Our goal in the present series was to evaluate the association of adjuvant interferon with RFS, distant metastasis-free survival (DMFS) and OS in a modern experience at a comprehensive melanoma clinical program focusing on accurate staging and aggressive efforts to achieve regional control in stage III melanoma.

\section{Methods \\ Patient selection \& histopathologic analyses}

After obtaining Institutional Review Board approval, we performed a retrospective review from 1998 to 2015 and identified 699 patients from an institutional database diagnosed with node-positive cutaneous melanoma without distant metastatic disease (Figure 1). After excluding patients who had all of their initial management of node-positive melanoma at outside institutions and those presenting with recurrent or metastatic disease $(\mathrm{n}=143)$, satellite or intransit metastasis only (American Joint Committee on Cancer [AJCC] 7th edition N2c; $n=4$ ), unknown recurrence status $(\mathrm{n}=99)$, unclear treatment records $(\mathrm{n}=17),<12$ months follow-up from the time of node dissection $(n=27)$ or noninterferon-adjuvant systemic therapy $(n=25), 385$ patients remained for analysis. All patients had AJCC stage III melanoma (any T, N1-3, M0) [17] and no clinical, radiologic, or pathologic evidence of metastasis beyond regional LNs. Only 7th edition AJCC staging was used. Patients without a known primary (T0, N1b-3, M0) were included. Demographic, histopathologic, radiation and outcome data were reviewed.

The patients were treated with either SLNB alone $(\mathrm{n}=47)$ or followed by completion lymph node dissection (LND; $\mathrm{n}=252)$ or therapeutic LND $(\mathrm{n}=86)$. The LND were evaluated using hematoxylin and eosin-stained sections. Serial sectioning and S100 and Melan A immunohistochemistry were used in the evaluation of all sentinel nodes. The patients were selected for radiotherapy based on high risk features for regional recurrence, such as any $\mathrm{LN}$ with extracapsular extension present, or $\geq 1$ involved parotid $\mathrm{LN}, \geq 2$ involved axillary LNs, $\geq 3$ involved groin $\mathrm{LNs}$, or at least one neck $\mathrm{LN} \geq 3 \mathrm{~cm}$ or axillary/groin $\mathrm{LN} \geq 4 \mathrm{~cm}$.

The patients who received regional radiation were treated with either electrons or photons to the postoperative regional nodal basin, with or without including the primary tumor site as indicated. Additional technical radiotherapy details were outlined in a previous manuscript [16]. 


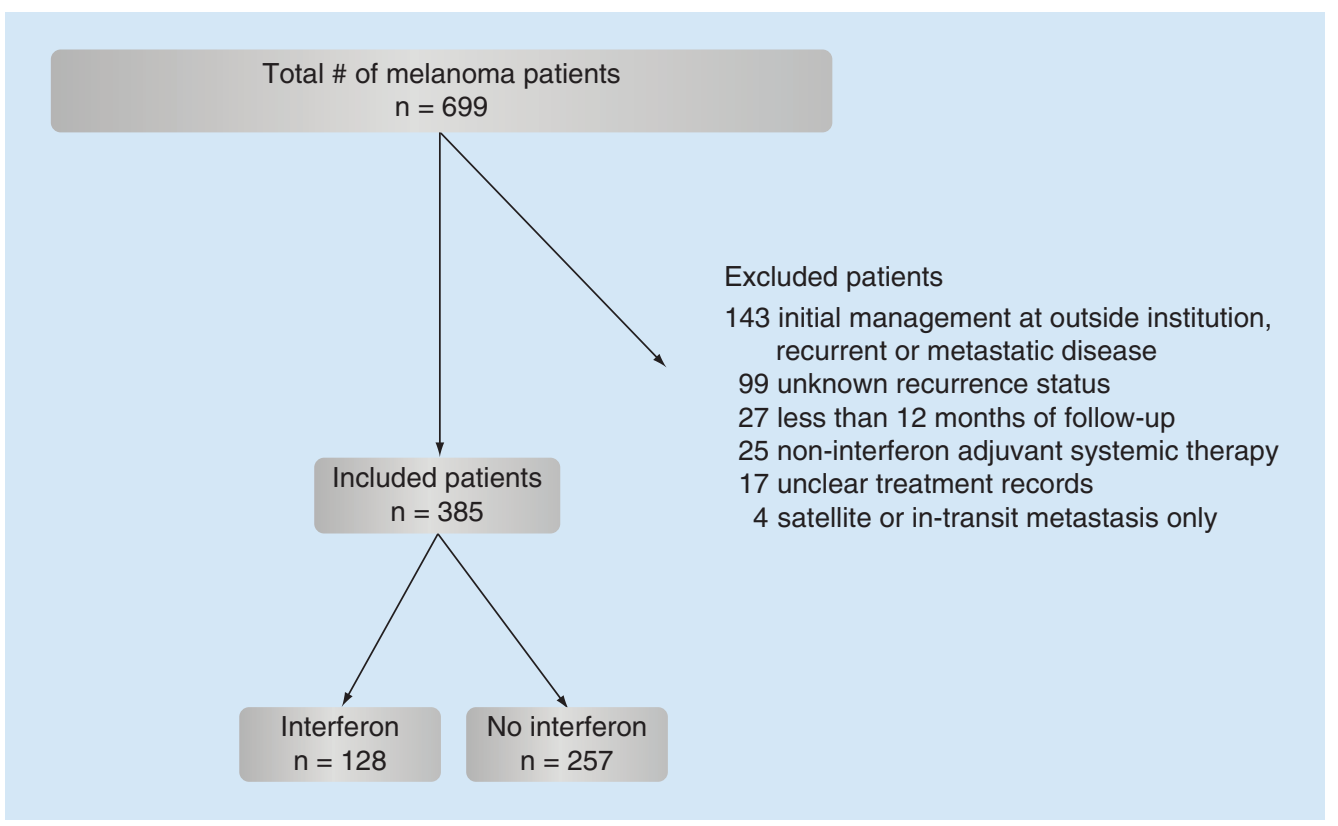

Figure 1. The flow chart on retrospective review of node-positive melanoma patients.

\section{Systemic therapy}

Delivery of adjuvant therapy commenced within 6 months of surgery and prior to recurrence at any site. Some form of adjuvant therapy was administered to 156 patients (38.0\%); adjuvant high-dose or pegylated interferon was given to 128 (82.1\% of patients receiving any adjuvant therapy). Of the 25 patients who received noninterferon adjuvant systemic therapy and were excluded from the analysis, their therapies included investigational vaccines $(\mathrm{n}=9)$, ipilimumab $(\mathrm{n}=7)$, BCG $(\mathrm{n}=7)$, chemotherapy or biochemotherapy $(\mathrm{n}=3)$ and an investigational CD40 agonist $(\mathrm{CP}-870,893 ; \mathrm{n}=2)$. Adjuvant interferon was delivered to 31 out of 86 patients $(36.0 \%)$ undergoing therapeutic LND and to 97 out of 299 patients (32.4\%) after SLNB \pm completion LND. Of the 128 patients receiving interferon, $75.8 \%$ had SLNB-detected disease. This is comparable to the proportion of patients with SLNB-detected disease within our study population as a whole (299 out of 385, 77.7\%). Adjuvant interferon was delivered to similar percentages of patients who did and did not receive radiation ( $22.7 \mathrm{vs} 19.8 \%$, respectively).

\section{Statistical analysis}

Statistical analysis was performed using SPSS ${ }^{\circledR}$ version 22.0 (IBM, IL, USA). The primary end points were RFS, defined as freedom from disease recurrence at any site or death, DMFS, defined as freedom from tumor spread beyond the regional LNs or death, and OS among all patients. Actuarial rates of RFS, DMFS and OS were calculated using the Kaplan-Meier method, and differences in rates based on individual variables were assessed with the log-rank test. All clinical, histopathologic and treatment variables were added to Cox multivariate analysis regression models. Wilcoxon rank-sum test was used to compare continuous variables, which were split using clinically meaningful cut-points, including a LN size of $\leq 2.0 \mathrm{vs}>2.0 \mathrm{~cm}$. All tests were two-sided and an $\alpha$ (type I) error $\leq 0.05$ was considered to be statistically significant.

\section{Results}

Patient, clinicopathologic \& treatment characteristics

Of 385 patients included in this analysis, the median age was 59 years (range 14-91 years). The patients were more likely to be male $(66.8 \%)$, and have $\mathrm{T} 3$ or T4 primary tumors (38.2 and 33.8\%, respectively), involved axillary LNs (57.4\%) and micrometastatic nodal disease (AJCC N1a/N2a, 56.4\%). Extranodal extension was present in $34.0 \%$ of patients. The postoperative regional radiation therapy was delivered to the involved nodal basin prior to recurrence in 80 cases $(20.8 \%$ ) to a median dose of 54 Gy (range 30-60 Gy) in 27 fractions (range 5-30). Clinicopathologic and treatment characteristics among patients treated with and without adjuvant interferon are 


\begin{tabular}{|c|c|c|c|c|c|}
\hline Characteristics & Classification & $\begin{array}{l}\text { All patients }(n=385), n \\
(\%)\end{array}$ & $\begin{array}{l}\text { Interferon }(n=128), n \\
(\%)\end{array}$ & $\begin{array}{l}\text { No adjuvant therapy ( } \\
=257), n(\%)\end{array}$ & p-value \\
\hline Median age (years, range) & & $59(14-91)$ & $50(14-80)$ & $65(14-91)$ & $<0.001$ \\
\hline \multirow[t]{2}{*}{ Sex } & Female & $128(33.2)$ & $44(34.4)$ & $84(32.7)$ & 0.74 \\
\hline & Male & $257(66.8)$ & $84(65.6)$ & $173(67.3)$ & - \\
\hline \multirow[t]{5}{*}{ Tumor T stage } & $\mathrm{T} 1$ & $11(2.9)$ & $4(3.1)$ & $7(2.7)$ & 0.58 \\
\hline & $\mathrm{T} 2$ & $62(16.1)$ & $24(18.8)$ & $38(14.8)$ & - \\
\hline & T3 & $147(38.2)$ & $42(32.8)$ & $105(40.9)$ & - \\
\hline & T4 & $130(33.8)$ & $47(36.7)$ & $83(32.3)$ & - \\
\hline & Tо & $35(9.1)$ & $11(8.6)$ & $24(9.3)$ & - \\
\hline \multirow[t]{3}{*}{ LND site } & Groin & $87(22.6)$ & $30(23.4)$ & $57(22.2)$ & 0.86 \\
\hline & Head and neck & $77(20)$ & $27(21.1)$ & $50(19.5)$ & - \\
\hline & Axilla & $221(57.4)$ & $71(55.5)$ & $150(58.4)$ & - \\
\hline Median LNs positive, $\mathrm{n}$ (range) & & $2(1-34)$ & $2(1-34)$ & $2(1-22)$ & 0.53 \\
\hline \multirow[t]{5}{*}{ Tumor $\mathrm{N}$ stage } & $\mathrm{N} 1 \mathrm{a} / \mathrm{N} 2 \mathrm{a}$ & $217(56.4)$ & $76(59.4)$ & $141(54.9)$ & 0.4 \\
\hline & $\mathrm{N} 1 \mathrm{~b} / \mathrm{N} 2 \mathrm{~b} / \mathrm{N} 3$ & $168(43.6)$ & $52(40.6)$ & $116(45.1)$ & - \\
\hline & N1 & $170(44.2)$ & $60(46.9)$ & $110(42.8)$ & 0.68 \\
\hline & N2 & $87(22.6)$ & $29(22.7)$ & $58(22.6)$ & - \\
\hline & N3 & $128(33.2)$ & $39(30.5)$ & $89(34.6)$ & - \\
\hline \multirow[t]{2}{*}{ Extranodal extension } & Absent & $254(66.0)$ & $84(65.6)$ & $170(66.1)$ & 0.92 \\
\hline & Present & $131(34.0)$ & $44(34.4)$ & $87(33.9)$ & - \\
\hline Median LN size, cm (range) & & $2.0(0.3-15)$ & $2.0(0.5-8.0)$ & $2.2(0.3-15.0)$ & 0.11 \\
\hline \multirow[t]{2}{*}{ Regional radiation therapy? } & No & $305(79.2)$ & $99(77.3)$ & $206(80.2)$ & 0.52 \\
\hline & Yes & $80(20.8)$ & $29(22.7)$ & $51(19.8)$ & - \\
\hline
\end{tabular}

shown in Table 1. Interferon was more frequently delivered to the patients who were younger (median 50 vs 65 years; $\mathrm{p}<0.001$ ), while no other clinicopathologic characteristics were different between treatment groups.

\section{Relapse-free survival}

Surviving patients had a minimum follow-up of 12 months, with a median follow-up of 70 months (range 13-180 months). There were $247(64.2 \%)$ relapses among the 385 patients, with a median time to relapse of 15 months. RFS, DMFS and OS outcomes, along with associated variables, are shown in Table 2. Multivariable analysis revealed male sex (hazard ratio [HR]: 1.62; 95\% CI: 1.22-2.15; $\mathrm{p}<0.001$ ), AJCC T3/4 tumor status (HR: 1.89; 95\% CI: 1.30-2.75; $\mathrm{p}=0.001$ ), AJCC N1b/N2b/N3 nodal stage (HR: 1.88; 95\% CI: 1.39-2.55; $\mathrm{p}<0.001$ ), extranodal extension (HR: 2.25; 95\% CI: 1.62-3.13; $\mathrm{p}<0.001$ ) and delivery of regional radiotherapy (HR: 2.05; 95\% CI: $1.37-3.08 ; \mathrm{p}=0.001$ ) as predictors of worse RFS.

Adjuvant interferon was associated with improved RFS on univariate (5-year estimate 40.2 vs $25.8 \%$; $p<0.001$ ) and multivariate analysis (HR: 0.55; 95\% CI: 0.42-0.74; $\mathrm{p}<0.001$ ) (Figure 2A).

\section{Distant metastasis-free survival}

In total, 129 patients (33.5\%) developed distant metastases. Median time to distant metastasis after surgery was 14 months. Multivariable analysis revealed male sex (HR: 1.54; 95\% CI: 1.16-2.05; $\mathrm{p}=0.003$ ), AJCC T3/4 tumor status (HR: 2.09; 95\% CI: 1.42-3.07; p < 0.001), AJCC N1b/N2b/N3 nodal stage (HR: 1.61; 95\% CI: 1.18-2.19; $\mathrm{p}=0.002$ ), extranodal extension (HR: 1.87; 95\% CI: 1.34-2.61; $\mathrm{p}<0.001$ ), nodal metastasis $>2 \mathrm{~cm}$ (HR: 1.39; 95\% CI: 1.04-1.86; $\mathrm{p}=0.027$ ) and delivery of regional radiotherapy (HR: 1.61; 95\% CI: 1.07-2.44; $\mathrm{p}=0.044)$ as predictors of worse DMFS.

Adjuvant interferon was associated with improved DMFS on univariate ( 5 -year estimate 47.9 vs $36 \% ; \mathrm{p}=0.003$ ) and multivariate analysis (HR: 0.59; 95\% CI: 0.44-0.79; $\mathrm{p}<0.001$ ) (Figure 2B). Of the 129 patients with distant 


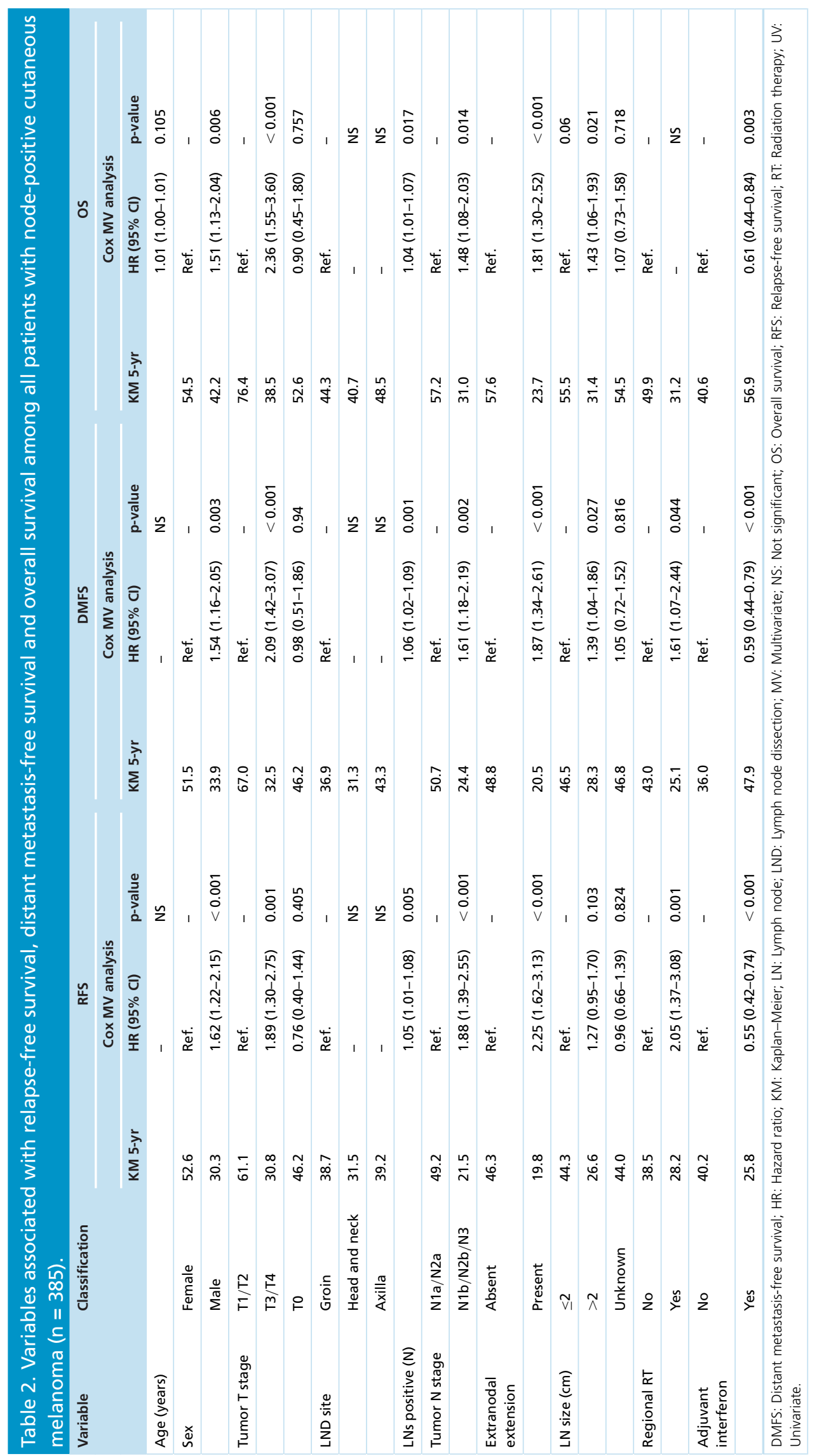



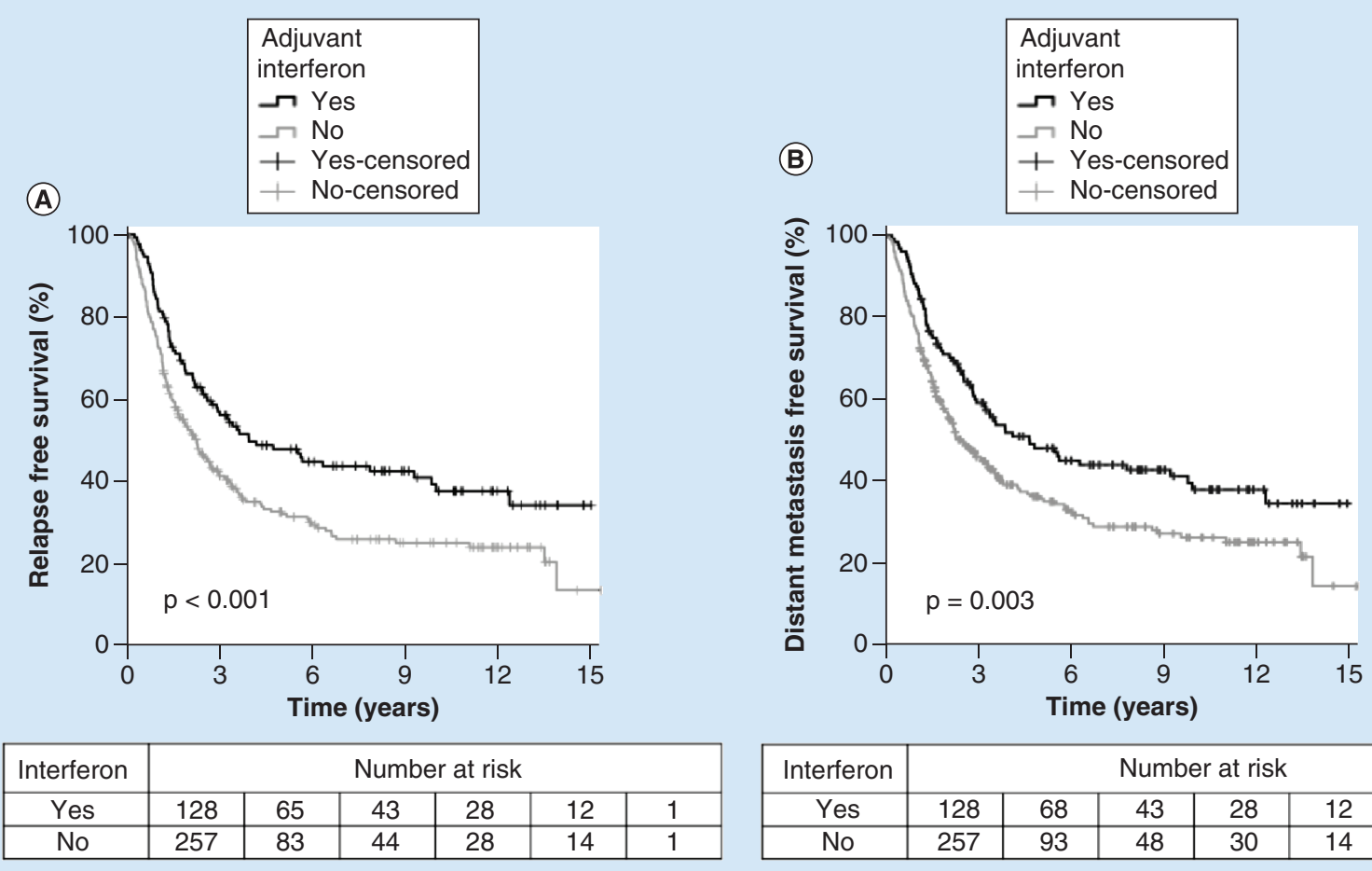

\begin{tabular}{|c|c|c|c|c|c|c|}
\hline Interferon & \multicolumn{6}{|c|}{ Number at risk } \\
\hline Yes & 128 & 68 & 43 & 28 & 12 & 1 \\
\hline No & 257 & 93 & 48 & 30 & 14 & 1 \\
\hline
\end{tabular}

(C)

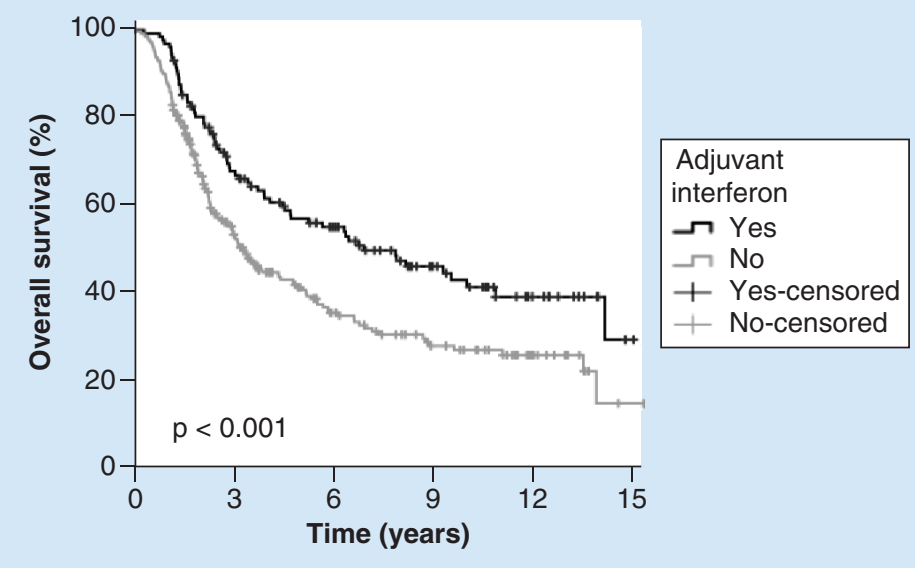

\begin{tabular}{|c|c|c|c|c|c|c|}
\hline Interferon & \multicolumn{6}{|c|}{ Number at risk } \\
\hline Yes & 128 & 79 & 54 & 31 & 13 & 1 \\
\hline No & 257 & 109 & 53 & 31 & 14 & 1 \\
\hline
\end{tabular}

Figure 2. Graphs of survival versus time.

failures, 114 (88.4\%) had died by last follow-up. In these cases, the median time from distant failure to death was 8 months (range, 15 days-97 months).

\section{Overall survival}

Of the 385 total patients in our study, $225(58.4 \%)$ had died by last follow-up. Median survival after surgery was 47 months. Multivariable analysis revealed male sex (HR: 1.51; 95\% CI: 1.13-2.04; p = 0.006), AJCC T3/4 tumor status (HR: 2.36; 95\% CI: 1.55-3.60; $\mathrm{p}<0.001$ ), AJCC N1b/N2b/N3 nodal stage (HR: 1.48; 95\% CI: 
1.08-2.03; $\mathrm{p}=0.014$ ), extranodal extension (HR: 1.81; 95\% CI: $1.30-2.52 ; \mathrm{p}<0.001$ ) and nodal metastasis size $>2 \mathrm{~cm}$ (HR: 1.43 ; 95\% CI: $1.06-1.93 ; \mathrm{p}=0.021$ ) as predictors of worse OS.

Adjuvant interferon was associated with improved OS on univariate (5-year estimate 56.9 vs $40.6 \%$; $\mathrm{p}<0.001$ ) and multivariate analysis (HR: 0.61; 95\% CI: 0.44-0.84; $\mathrm{p}=0.003$ ) (Figure 2C).

Age-matched analysis

To determine if our results might be explained by selection of younger patients, an exploratory age-matched comparison of patients treated with $(n=67$, median 61 years $)$ and without $(n=233$, median 63 years) adjuvant interferon was conducted. For age-matched patients, treatment with interferon continued to show improved RFS (5-year estimate 43.8 vs 33.7\%; HR: 0.53; 95\% CI: 0.37-0.77; $\mathrm{p}=0.001$ ), DMFS (5-year estimate: 43.9 vs 37.5\%; HR: 0.57; 95\% CI: 0.39-0.83; $\mathrm{p}=0.004)$ and OS (5-year estimate: 57.3 vs 42.5\%; HR: 0.53; 95\% CI: 0.35-0.78; $\mathrm{p}=0.001)$ on multivariate analysis. Figure 3 shows Kaplan-Meier plots for these respective end points.

\section{Discussion}

Many years of research have been devoted toward the discovery of systemic agents that could improve survival while minimizing toxicity in the adjuvant treatment of stage III cutaneous melanoma. Prior to the introduction of CTLA-4 blockade as adjuvant therapy, interferon was the best-studied and main choice in systemic therapy for the patients with stage III melanoma. Currently, the NCCN guidelines for stage III melanoma state that adjuvant interferon can be given to delay recurrence, but that 'there is no impact on overall survival' [12]. Various forms, duration and doses of interferon have been employed in studies, either alone or in combination with other systemic agents, with no regimen or formulation proven superior [6-8,18-22]. RFS has been the primary end point in most of these trials. Although the benefit in RFS, confirmed in this series, has remained consistent, any OS advantage with interferon is controversial $[9,23,24]$. Due to the combination of its cost, toxicity profile and presumed marginal OS benefit, interferon has fallen out of favor among oncologists in recent years. Our findings, however, show a substantial and clinically relevant improvement in both DMFS and OS with delivery of adjuvant interferon in stage III melanoma patients treated at a single center with an aggressive approach to staging and regional disease control.

Nodal tumor burden significantly affects prognosis [3], and the typical node-positive patient seen today will likely have a metastatic tumor burden of $1 \mathrm{~mm}$ or less within a single LN, compared with the predominant bulky disease seen in the adjuvant trials that resulted in the approval of high-dose or pegylated interferon $[6,19]$. In concordance with this trend, the majority of patients $(77.7 \%)$ in our study had nodal disease detected by SLNB. Of note, the number needed to treat is approximately six patients (95\% CI: 3-31 patients); compared with a number needed to treat of 29 patients in the meta-analysis of Mocellin et al. [9].

There is much excitement surrounding the advances made with CTLA-4 blockade and its increasingly prominent role in the treatment of melanoma. Improvements in survival were seen with the checkpoint inhibitor ipilimumab in Phase III trials [13,14]. A sustained OS benefit in metastatic melanoma and later in the adjuvant setting led to US FDA approval of ipilimumab for these roles in 2011 [13] and 2015, respectively [14]. The EORTC 18071 trial compared ipilimumab at $10 \mathrm{mg} / \mathrm{kg}$ with placebo in high-risk stage III patients who had previous complete LND [14]. The patients with greatest tumor burden experienced the most benefit, while stage IIIA patients did not show significant improvement with therapy. Comparing the HR for ipilimumab versus placebo to interferon versus no adjuvant therapy in our study, interferon compares favorably in RFS ( 0.76 vs 0.55 , respectively), DMFS ( 0.76 vs 0.59$)$ and OS (0.72 vs 0.61 ) [14]. Furthermore, the rates of grade 3,4 and even grade 5 toxicity with ipilimumab in the EORTC trial were concerning, with approximately $40 \%$ of patients discontinuing treatment by the end of the first four doses. Although treatment with interferon can have significant side effects, not all patients experience these, and most patients can complete a year of therapy. Additionally, while the patients undergoing adjuvant interferon usually return fairly quickly to their baseline function, there appears to be less/slower resolution in toxicity following treatment with ipilimumab [25]. As ipilimumab was compared with placebo in EORTC 18071, the results of a US Intergroup Phase III trial, ECOG 1609, comparing ipilimumab with high-dose interferon, will be of key importance in future decision-making regarding adjuvant therapy. More recently, results of CheckMate 238 showed improved RFS at 18 months with 1 year of anti-PD1 therapy when compared with ipilimumab (66.4 vs $52.7 \%$, respectively) in patients with resected Stage IIIB, IIIC or IV melanoma [15]. Anti-PD1 therapy appears to have an improved therapeutic index over any previous adjuvant systemic therapy; while longer follow-up is required 

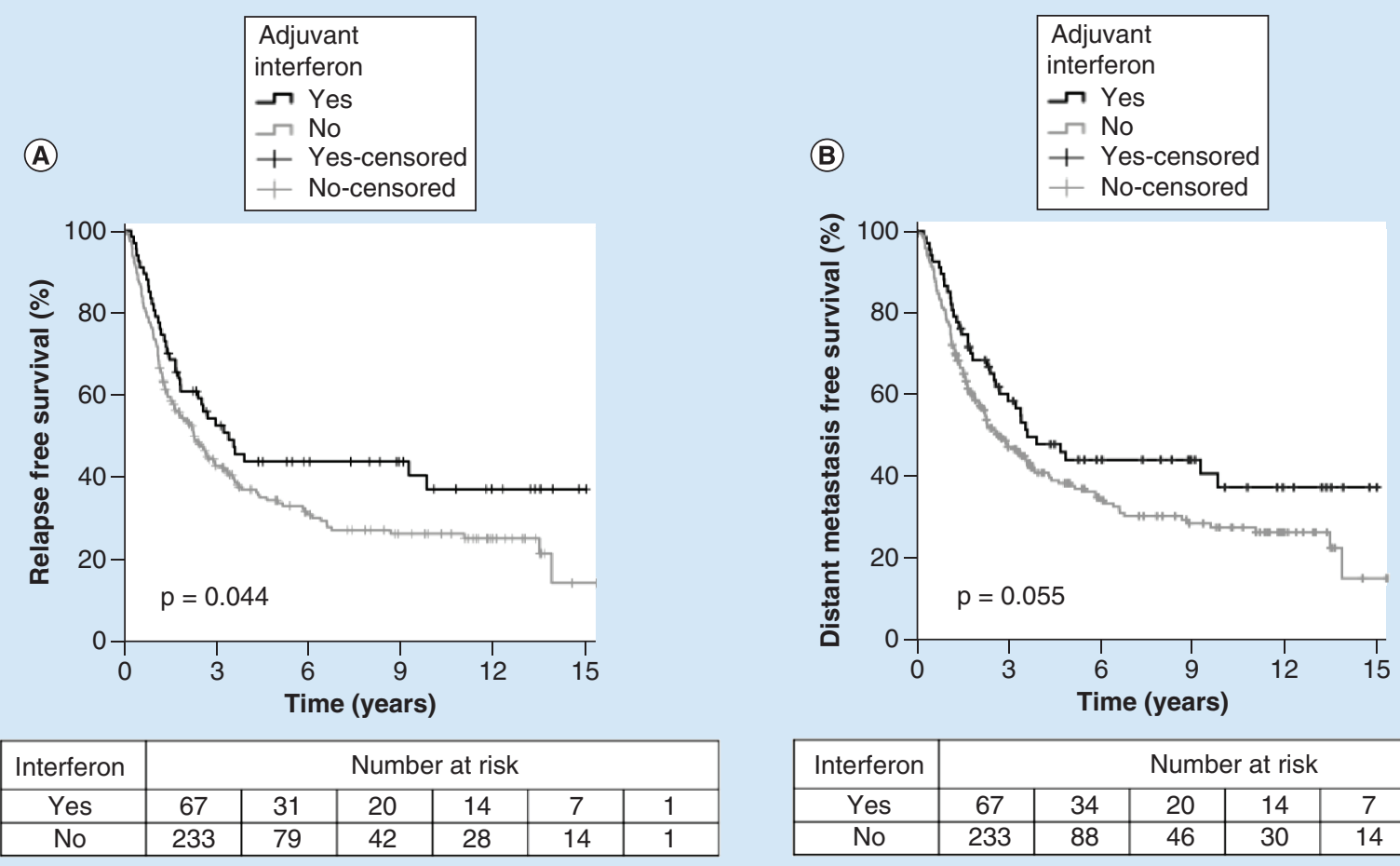

\begin{tabular}{|c|c|c|c|c|c|c|}
\hline Interferon & \multicolumn{7}{|c|}{ Number at risk } \\
\hline Yes & 67 & 34 & 20 & 14 & 7 & 1 \\
\hline No & 233 & 88 & 46 & 30 & 14 & 1 \\
\hline
\end{tabular}

(C)

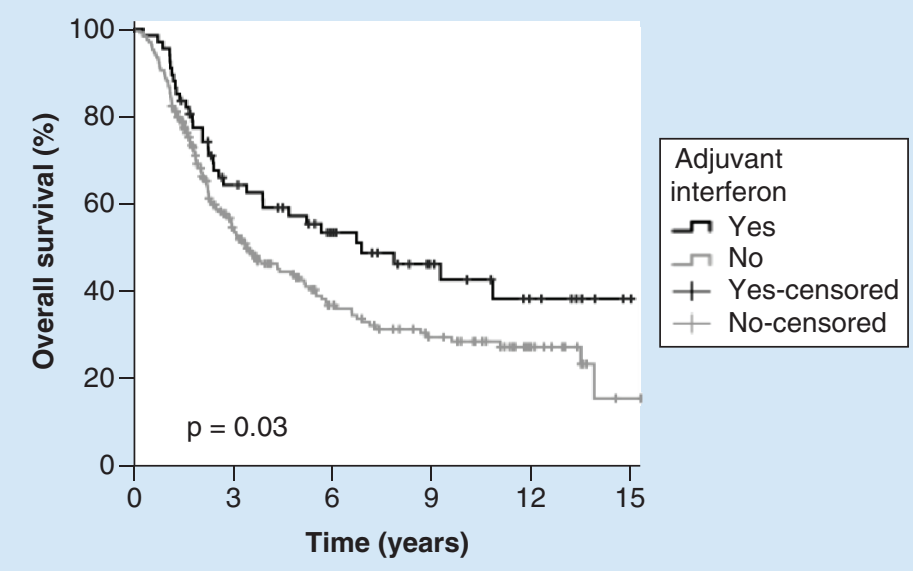

\begin{tabular}{|c|c|c|c|c|c|c|}
\hline Interferon & \multicolumn{6}{|c|}{ Number at risk } \\
\hline Yes & 67 & 38 & 25 & 14 & 7 & 1 \\
\hline No & 233 & 103 & 50 & 31 & 14 & 1 \\
\hline
\end{tabular}

Figure 3. Graphs of age-matched survival versus time.

to demonstrate survival impact and results of trials comparing checkpoint blockade to interferon are awaited, the CheckMate 238 trial is widely considered to have established a new standard of care for these patients [26].

Limitations of this study include its retrospective, nonrandomized nature and the long timeframe over which it took place. Data for calculating melanoma-specific survival were not available to allow us to analyze death from competing causes. Although LN size of $2 \mathrm{~cm}$ was used in our primary analysis, other cut points could be used for stratification in future studies. Another limitation was the inclusion of standard high-dose and pegylated versions of interferon together within the adjuvant interferon group. Despite these limitations, the eligibility criteria for this series ensured that each patient had consistent management and reasonable follow-up. The finding of improved 
survival outcomes with adjuvant interferon within the setting of a modern, aggressive approach to staging and treatment is noteworthy even with the advances that have occurred in the management of metastatic melanoma. The adjuvant therapy landscape in melanoma is rapidly changing, and in the USA, adjuvant anti-PD1 therapy has emerged as the current standard despite no prospective clinical trial data yet available to show it conveys a survival benefit to patients compared with interferon or ipilimumab. Clinical trials comparing interferon to ipilimumab and anti-PD1 in the adjuvant setting (E1609 and S1404, respectively) have been conducted, and we await mature results.

\section{Conclusion}

Adjuvant interferon was associated with improved RFS, DMFS and OS at our center, when used as part of an aggressive approach to staging and treatment of stage III melanoma. This benefit remained apparent on age-matched analysis. This single-institution experience with adjuvant interferon provides additional context for comparisons with anti-PD1, and may be of particular relevance in the international arena, where adjuvant anti-PD1 or antiCTLA4 therapy has not yet achieved widespread adoption.

\section{Financial \& competing interests disclosure}

VK Sondak: Consultant/Advisory Board/DSMB: Amgen, BMS, Merck, Novartis, Provectus; JS Zager: Consultant/Advisory Board: Amgen, Castle Biosciences, Delcath Systems, Philochem. Research support from Amgen, Provectus, Castle, Delcath, Philochem. NI Khushalani: Consultant/Advisory Board/DSMB: BMS, Castle Biosciences, EMD Serono, Astra Zeneca, Genentech. Stock Ownership: Bellicum Phamaceuticals. Research support from NCCN, BMS, Merck, HUYA Biosciences, Pfizer, Eisai, Threshold, Tracon, GlaxoSmithKline, Novartis. The authors have no other relevant affiliations or financial involvement with any organization or entity with a financial interest in or financial conflict with the subject matter or materials discussed in the manuscript apart from those disclosed.

No writing assistance was utilized in the production of this manuscript.

\section{Ethical conduct of research}

The authors state that they have obtained appropriate institutional review board approval or have followed the principles outlined in the Declaration of Helsinki for all human or animal experimental investigations.

\section{Open access}

This work is licensed under the Attribution-NonCommercial-NoDerivatives 4.0 Unported License. To view a copy of this license, visit http://creativecommons.org/licenses/by-nc-nd/4.0/

\section{References}

1. Guy GP Jr, Thomas CC, Thompson T, Watson M, Massetti GM, Richardson LC. Vital signs: melanoma incidence and mortality trends and projections - United States, 1982-2030. MMWR Morb. Mortal. Wkly Rep. 64(21), 591-596 (2015).

2. Balch CM, Gershenwald JE, Soong SJ et al. Multivariate analysis of prognostic factors among 2313 patients with stage III melanoma: comparison of nodal micrometastases versus macrometastases. J. Clin. Oncol. 28(14), 2452-2459 (2010).

3. Van Der Ploeg AP, Van Akkooi AC, Rutkowski P et al. Prognosis in patients with sentinel node-positive melanoma is accurately defined by the combined Rotterdam tumor load and Dewar topography criteria. J. Clin. Oncol. 29(16), 2206-2214 (2011).

4. Morton DL, Thompson JF, Cochran AJ et al. Sentinel-node biopsy or nodal observation in melanoma. N. Engl. J. Med. 355(13), 1307-1317 (2006).

5. Garbe C, Radny P, Linse R et al. Adjuvant low-dose interferon \{alpha\}2a with or without dacarbazine compared with surgery alone: a prospective-randomized Phase III DeCOG trial in melanoma patients with regional lymph node metastasis. Ann. Oncol. 19(6), 1195-1201 (2008).

6. Kirkwood JM, Strawderman MH, Ernstoff MS, Smith TJ, Borden EC, Blum RH. Interferon alfa-2b adjuvant therapy of high-risk resected cutaneous melanoma: the Eastern Cooperative Oncology Group Trial EST 1684. J. Clin. Oncol. 14(1), 7-17 (1996).

7. Kirkwood JM, Ibrahim JG, Sondak VK et al. High- and low-dose interferon alfa-2b in high-risk melanoma: first analysis of intergroup trial E1690/S9111/C9190. J. Clin. Oncol. 18(12), 2444-2458 (2000).

8. Kirkwood JM, Ibrahim JG, Sosman JA et al. High-dose interferon alfa-2b significantly prolongs relapse-free and overall survival compared with the GM2-KLH/QS-21 vaccine in patients with resected stage IIB-III melanoma: results of intergroup trial E1694/S9512/C509801. J. Clin. Oncol. 19(9), 2370-2380 (2001).

9. Mocellin S, Pasquali S, Rossi CR, Nitti D. Interferon alpha adjuvant therapy in patients with high-risk melanoma: a systematic review and meta-analysis. J. Natl Cancer Inst. 102(7), 493-501 (2010). 
10. Eggermont AM, Suciu S, Testori A et al. Ulceration and stage are predictive of interferon efficacy in melanoma: results of the Phase III adjuvant trials EORTC 18952 and EORTC 18991. Eur. J. Cancer 48(2), 218-225 (2012).

11. Kirkwood JM, Ibrahim J, Lawson DH et al. High-dose interferon alfa-2b does not diminish antibody response to GM2 vaccination in patients with resected melanoma: results of the Multicenter Eastern Cooperative Oncology Group Phase II Trial E2696. J. Clin. Oncol. 19(5), 1430-1436 (2001).

12. Coit DG, Thompson JA, Algazi A et al. Melanoma, Version 2.2016, NCCN Clinical Practice Guidelines in Oncology. J. Natl Compr. Cancer Netw. 14(4), 450-473 (2016).

13. Hodi FS, O’Day SJ, McDermott DF et al. Improved survival with ipilimumab in patients with metastatic melanoma. $N$. Engl. J. Med. 363(8), 711-723 (2010).

14. Eggermont AM, Chiarion-Sileni V, Grob JJ et al. Prolonged survival in stage III melanoma with ipilimumab adjuvant therapy. N. Engl. J. Med. 375(19), 1845-1855 (2016).

15. Weber J, Mandala M, Del Vecchio M et al. Adjuvant nivolumab versus ipilimumab in resected stage III or IV melanoma. N. Engl. J. Med. 377(19), 1824-1835 (2017).

16. Strom T, Torres-Roca JF, Parekh A et al. Regional radiation therapy impacts outcome for node-positive cutaneous melanoma. J. Natl Compr. Canc. Netw. 15(4), 473-482 (2017).

17. Edge SB, Byrd DR, Compton CC, Fritz AG, Greene FL, Trotti A. AJCC Cancer Staging Manual (7th Ed.). Springer, NY, USA (2010).

18. Eggermont AM, Suciu S, Mackie R et al. Post-surgery adjuvant therapy with intermediate doses of interferon alfa $2 \mathrm{~b}$ versus observation in patients with stage IIb/III melanoma (EORTC 18952): randomized controlled trial. Lancet 366(9492), 1189-1196 (2005).

19. Eggermont AM, Suciu S, Santinami M et al. Adjuvant therapy with pegylated interferon alfa-2b versus observation alone in resected stage III melanoma: final results of EORTC 18991, a randomized Phase III trial. Lancet 372(9633), 117-126 (2008).

20. Hauschild A, Weichenthal M, Rass K et al. Efficacy of low-dose interferon \{alpha\}2a 18 versus 60 months of treatment in patients with primary melanoma of $>=1.5 \mathrm{~mm}$ tumor thickness: results of a randomized Phase III DeCOG trial. J. Clin. Oncol. 28(5), 841-846 (2010).

21. Cascinelli N, Belli F, Mackie RM, Santinami M, Bufalino R, Morabito A. Effect of long-term adjuvant therapy with interferon alpha-2a in patients with regional node metastases from cutaneous melanoma: a randomized trial. Lancet 358(9285), 866-869 (2001).

22. Pehamberger H, Soyer HP, Steiner A et al. Adjuvant interferon alfa-2a treatment in resected primary stage II cutaneous melanoma. Austrian Malignant Melanoma Cooperative Group. J. Clin. Oncol. 16(4), 1425-1429 (1998).

23. Lens MB, Dawes M. Interferon alfa therapy for malignant melanoma: a systematic review of randomized controlled trials. J. Clin. Oncol. 20(7), 1818-1825 (2002).

24. Wheatley K, Ives N, Hancock B, Gore M, Eggermont A, Suciu S. Does adjuvant interferon-alpha for high-risk melanoma provide a worthwhile benefit? A meta-analysis of the randomized trials. Cancer Treat. Rev. 29(4), 241-252 (2003).

25. Linardou H, Gogas H. Toxicity management of immunotherapy for patients with metastatic melanoma. Ann. Transl. Med. 4(14), 272 (2016).

26. Weber JS, Hodi FS, Wolchok JD et al. Safety profile of nivolumab monotherapy: a pooled analysis of patients with advanced melanoma. J. Clin. Oncol. 35(7), 785-792 (2017). 